\title{
Cord blood transferrin receptors to assess fetal iron status
}

\author{
David G Sweet, Geraldine A Savage, Richard Tubman, Terence R J Lappin,
} Henry L Halliday

\begin{abstract}
Objective-To study iron status at different gestational ages using cord blood serum transferrin receptors (STfRs).

Methods-STfRs, iron, ferritin, total iron binding capacity, haemoglobin, and reticulocytes were measured in 144 cord blood samples. The babies were divided into three groups according to gestation (26 very preterm (24-29 weeks); 50 preterm (30-36 weeks); 68 term (37-41 weeks)).

Results-Serum iron, ferritin, and total iron binding capacity were highest at term, whereas reticulocytes were highest in the very preterm. STfR levels were not influenced by gestation. Haemoglobin $(r=0.46 ; \mathrm{p}<0.0001)$ and reticulocytes $(r=0.42 ; \mathrm{p}<0.0001)$ were the only indices that independently correlated with STfR levels.

Conclusions-STfR levels in cord blood are not directly influenced by gestation and probably reflect the iron requirements of the fetus for erythropoiesis.

(Arch Dis Child Fetal Neonatal Ed 2001;85:F46-F48)
\end{abstract}

Keywords: erythropoiesis; iron metabolism; reticulocytes; transferrin receptors

Measurement of serum transferrin receptors provides a new method of assessing iron status, but there are few published data on their role in the fetus or neonate. Intracellular iron uptake is mediated through transferrin receptors on the cell membrane. ${ }^{1}$ These bind and internalise the transferrin-iron complex, release the iron into the cytosol, and return the transferrin molecule to the circulation. ${ }^{2}$ When intracellular iron is low, the synthesis of transferrin receptors is increased and ferritin is decreased enabling the cell simultaneously to mobilise iron stores and compete for iron from the circulation. ${ }^{3}$ Transferrin receptors can be measured in serum (serum transferrin receptor; STfR), and their concentration is related to the total amount of cell membrane bound transferrin receptor. ${ }^{4}$ The ratio of STfR to the log of the serum ferritin concentration (TfR-F index) is also used as a measure of tissue iron status, as this reflects requirement for iron in relation to available iron. $^{56}$

Iron depletion is unusual in the term newborn as the fetus is efficient at extracting iron even in the face of maternal iron depletion. ${ }^{78}$ Despite this, the STfR levels in cord and neonatal blood are higher than in adults, ${ }^{9-11}$ and levels in preterm babies increase further after treatment with erythropoietin. ${ }^{12}$
We hypothesised that in fetal life when iron is freely available the STfR levels would predominantly be influenced by the rate of erythropoiesis. We planned to assess what factors influenced cord blood STfR levels and to study fetal iron status at various gestational ages.

\section{Methods}

This was a prospective study of babies born in the Royal Maternity Hospital, Belfast between December 1997 and April 1999. The research ethics committee of the Queen's University of Belfast gave ethical approval for the study. Samples were collected from consecutive deliveries when a research fellow was available. Babies with haemolytic disease and pregnancies complicated by maternal diabetes mellitus were excluded. The babies were divided into groups according to their gestational age at birth, estimated by the duration of amenorrhoea combined with early ultrasound findings: very preterm (24-29 weeks); preterm (30-36 weeks); term (37-41 weeks). After delivery and maternal consent, umbilical venous blood (2-3 $\mathrm{ml}$ ) was taken from the placenta.

Each sample was divided into two aliquots. An EDTA sample $(0.6 \mathrm{ml})$ was sent immediately for an automated full blood count $\mathrm{CHb}$ and reticulocytes; Sysmex SE 9000 series analyser, Sysmex UK, Milton Keynes, Bedfordshire, UK). The remainder was left to clot at room temperature, then centrifuged, and the serum separated and stored at $-70^{\circ} \mathrm{C}$ for subsequent analysis. The concentrations of iron, ferritin, and STfR and the total iron binding capacity (TIBC) were measured. Iron was measured by the tripyridyltriazine method on a Technicon AAII AutoAnalyzer. TIBC was measured by mixing the sample with excess ferric ions which bind to unoccupied sites on transferrin. The unbound ferric ion was then removed by passage through an alumina column, and the remaining (bound) iron measured by the tripyridyltriazine method. An immunoradiometric assay using mouse antibody (ICN Pharmaceuticals) was used to measure ferritin. STfR was measured using an immunoenzymometric assay (Orion Diagnostica).

\section{STATISTICAL ANALYSIS}

Analysis of variance or the Kruskall-Wallis test was used where appropriate to test for significance of differences between gestational age groups. Spearman correlation coefficients were used to test significance of associations between indices in all of the samples. Multiple regression analysis using STfR as the dependent variable was used to assess the influence of 
Table 1 Cord blood iron status at various gestational ages

\begin{tabular}{lllll}
\hline Variable & $\begin{array}{l}\text { Very preterm } \\
(24-29 \text { weeks })\end{array}$ & $\begin{array}{l}\text { Preterm } \\
(30-36 \text { weeks })\end{array}$ & $\begin{array}{l}\text { Term } \\
(37-41 \text { weeks })\end{array}$ & p Value \\
\hline Number & 26 & 50 & 68 & \\
Gestation (weeks) & $27.1(1.6)$ & $32.8(1.9)$ & $39.0(1.3)$ & \\
Weight $(\mathrm{g})$ & $992(305)$ & $1904(555)$ & $3338(512)$ & \\
Hb $(\mathrm{g} / \mathrm{l})$ & $163(16)$ & $158(21)$ & $161(17)$ & $0.39^{\star}$ \\
Reticulocyte $(\%)$ & $4.7(1.7)$ & $3.9(1.3)$ & $3.4(0.8)$ & $<0.0001^{\star}$ \\
Iron $(\mu \mathrm{mol} / \mathrm{l})$ & $17.4(10.7)$ & $20.8(8.8)$ & $26.0(7.7)$ & $<0.0001^{\star}$ \\
TIBC $(\mu \mathrm{mol} / \mathrm{l})$ & $31.0(27.0-35.0)$ & $36.0(31.5-44.0)$ & $42.0(36.0-49.5)$ & $0.01 \dagger$ \\
Ferritin $(\mu \mathrm{g} / \mathrm{l})$ & $75(44-117)$ & $90(45-142)$ & $131(90-238)$ & $<0.0001 \dagger$ \\
STfR $(\mathrm{mg} / \mathrm{l})$ & $10.3(7.5-16.5)$ & $8.2(5.4-12.3)$ & $8.4(6.4-10.6)$ & $0.08 \dagger$ \\
TfR-F index & $5.5(3.3-8.6)$ & $4.6(2.8-7.1)$ & $3.8(3.0-4.9)$ & $0.01 \dagger$ \\
\hline
\end{tabular}

Normally distributed data shown as mean (SD), non-normal data as median (interquartile range).

${ }^{\star}$ Analysis of variance; $\dagger$ Kruskal Wallis.

$\mathrm{Hb}$, haemoglobin; TIBC, total iron binding capacity; STfR, serum transferrin receptor.

gestation, birth weight, and all of the other indices of iron and haematological status.

\section{Results}

A total of 144 cord blood samples were obtained from babies ranging from 24 to 41 weeks gestation. Forty two of the EDTA samples clotted and were unsuitable for full blood count. Ferritin, TIBC, and STfR were not normally distributed. Table 1 shows the mean gestation and birth weight and the distribution of the haematological indices in the three groups. Ferritin, iron, and TIBC were highest at term. Reticulocytes were highest in the very preterm babies and decreased towards term. Hb remained fairly constant throughout gestation. STfR levels were slightly higher in the very preterm group but this was not statistically significant. The TfR-F index decreased towards term.

Cord blood STfR correlated positively with $\mathrm{Hb}$ (fig 1) and reticulocyte count (fig 2) and weakly negatively with ferritin $(r=-0.23$; $\mathrm{p}=0.007)$ and iron $(r=-0.25 ; \mathrm{p}=0.003)$. Increasing gestational age was associated with decreasing STfR $(r=-0.16 ; \mathrm{p}=0.047)$ and reticulocytes $(r=-0.36 ; \mathrm{p}=0.0004)$, no

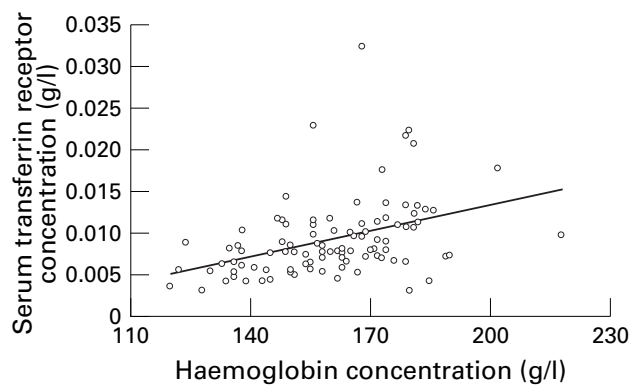

Figure 1 Correlation between levels of serum transferrin receptor and haemoglobin $(r=0.46 ; p<0.0001)$.

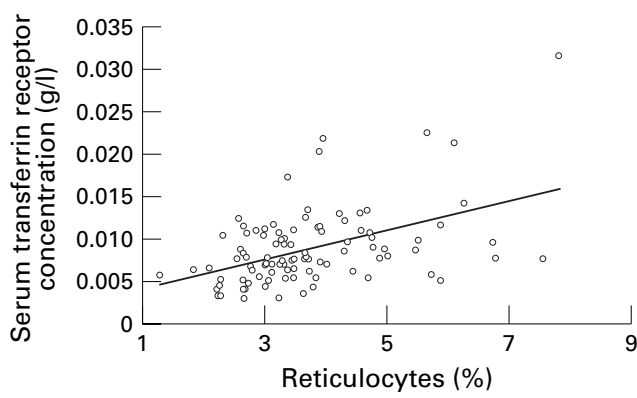

Figure 2 Correlation between serum transferrin receptor concentration and reticulocyte count $(r=0.42 ; p<0.0001)$. change in $\mathrm{Hb} \quad(r=0.046 ; \mathrm{p}=0.65)$, and increasing iron $(r=0.33 ; \mathrm{p}<0.0001)$, ferritin $(r=0.38 ; \mathrm{p}<0.0001)$, and TIBC $(r=0.38$; $\mathrm{p}=0.0003)$. Multiple regression analysis showed that $\mathrm{Hb}(\mathrm{p}=0.0011)$ and reticulocytes $(\mathrm{p}=0.0013)$ were the only variables independently associated with STfR levels.

\section{Discussion}

Fetal iron stores are accumulated at the end of the third trimester in preparation for birth and there is usually sufficient ferritin to meet the needs for growth over the first few months of life. ${ }^{13}$ Our data confirm this, with the highest iron and ferritin concentrations being found in the babies closest to term. Preterm delivery predisposes to subsequent development of iron deficiency because of the reduced transfer of iron, but the fetus should not have signs of iron deficiency at the time of delivery. ${ }^{14}$ Although cord blood ferritin is lower and STfR higher in the preterm baby, this should not be interpreted as iron deficiency but rather in the context of what is happening in intrauterine life.

After delivery, there is a rise in serum ferritin that either results from haemolysis or an acute phase response. ${ }^{15}$ Cord blood ferritin levels in this study are roughly one third of those we have reported previously for preterm babies during the first three days of life. ${ }^{16}$ Iron levels are about twice as high in cord blood as in samples taken during the first three days of life, and this may be explained by the fact that iron is being transferred to the fetus from the mother through the umbilical vein. ${ }^{16}$

Cord blood reticulocytes are highest in very preterm infants corresponding to the period of maximal fetal growth. This expansion of the red cell mass is required to keep pace with the growing fetus and it is logical that increased iron will be required during this time. STfR is positively correlated with reticulocytes and haemoglobin concentration, suggesting that the rise in transferrin receptors permits an increase in fetal red cell mass. Iron stores are not required during fetal life, and lower ferritin levels in the preterm infant do not give a meaningful indication of the iron status before delivery. Our findings are similar to those of Kuiper-Kramer et al ${ }^{9}$ showing only a weak correlation between measures of iron status in cord blood and STfR levels. Rusia et al ${ }^{11}$ have suggested that cord blood STfR levels could also be influenced by maternal iron status. This is unlikely as the fetus can usually extract enough iron to meet its requirements even when the mother has iron deficiency. It is worth noting that the babies born to anaemic mothers in their study were also lighter and had a higher cord blood $\mathrm{Hb}$ than those born to non-anaemic mothers. ${ }^{11}$ This may explain the higher cord blood STfR levels in this group.

At present, there is no reliable yardstick of iron status in the newborn infant, and it is not clear when or how much iron supplement should be given to the growing preterm baby. ${ }^{17}{ }^{18}$ STfR levels in cord blood appear to be predominantly influenced by the rate of erythropoiesis in utero. After delivery, they are also influenced by the rate of erythropoiesis, 
but it is not clear to what extent they are affected by iron deficiency as well. ${ }^{12}$ Further longitudinal studies of STfR in growing preterm babies are required to determine the relative contribution of inadequate erythropoiesis or iron deficiency to the development of anaemia of prematurity.

We conclude that STfR levels in cord blood reflect the fetal iron requirements for erythropoiesis and are not directly influenced by gestation. These requirements are slightly higher during the period of rapid fetal growth in the early third trimester than at term.

This project was supported by a grant from the Northern Ireland Mother and Baby Appeal.

1 Cook JD, Skikne BS, Baynes RD. Serum transferrin receptor. Anпu Rev Med 1993;44:63-74.

2 Feelders RA, Kuiper-Kramer EPA, van Eijk HG. Structure, function and clinical significance of transferrin receptors. Clinical Chemistry and Laboratory Medicine 1999;37:1-10.

3 Klausner RD, Rouault TA, Harford JB. Regulating the fate of mRNA: the control of cellular iron metabolism. Cell 1993; 72:19-28.

4 Shih YJ, Baynes RD, Hudson BG, Flowers CH, Skikne BS, Cook JD. Serum transferrin receptor is a truncated form of tissue receptor. F Biol Chem 1990;265:19077-81.

5 Skikne BS, Flowers CH, Cook JD. Serum transferrin receptor: a quantitative measure of tissue iron deficiency. Blood 1990;75:1870-6.

6 Punnonen K, Irjala K, Rajamaki A. Serum transferrin receptor and its ratio to serum ferritin in the diagnosis of receptor and its ratio to serum ferritin in
iron deficiency. Blood 1997;89:1052-7.
7 Siimes ASI, Siimes MA. Changes in the concentration of ferritin in the serum during fetal life in singletons and twins. Early Hum Dev 1986;13:47-52.

8 Lao TT, Loong EPL, Chin RKH, Lam CWK, Lam YM. Relationship between newborn and maternal iron status and haematological indices. Biol Neonate 1991;60:303-7.

9 Kuiper-Kramer EP, Baerts W, Bakker R, van Eyck J, van Raan J, van Eijk HG. Evaluation of the iron status of the newborn by soluble transferrin receptors in serum. Clinical Chemistry and Laboratory Medicine 1998;36:17-21.

10 Carpani G, Buscaglia M, Ghishoni L, et al. Soluble transferrin receptor in the study of fetal erythropoietic activity. $\mathrm{Am}$ f Hematol 1996;52:192-6.

11 Rusia U, Flowers C, Madan N, Agarwal N, Sood SK, Sikka M. Serum transferrin receptor levels in the evaluation of iron deficiency in the neonate. Acta Paediatr fpn 1996;38:455-9.

12 Kivivuori SM, Heikinheimo M, Teppo AM, Siimes MA. Early rise in serum concentration of transferrin receptor induced by recombinant human erythropoietin in verylow-birth weight-infants. Pediatr Res 1994;36:85-9.

13 Lozoff B, Jiminez E, Wolff AB. Long term developmental outcome of infants with iron deficiency. $N$ Engl $7 \mathrm{Med}$ 1991;325:687-94

14 Schulman I. The anemia of prematurity. $f$ Pediatr 1959;54:663-72.

15 Haga P. Plasma ferritin concentrations in preterm infants in cord blood and during the early anaemia of prematurity. Acta Paediatr Scand 1980;69:637-41.

16 Halliday HL, Lappin TRJ, McClure G. Iron status of the preterm infant during the first year of life. Biol Neonate 1984;45:228-35.

17 Halliday HL, Lappin TRJ, McClure BG. Do all preterm infants need iron supplements? Ir Med F 1983;76:430-2.

18 Griffin IJ, Cooke RJ, Reid MM, McCormick KPB, Smith JS. Iron nutritional status in preterm infants fed formulas fortified with iron. Arch Dis Child Fetal Neonatal Ed 1999;81:F45-9. 